\title{
Investigation of Thermal Inhomogeneities in Liquid Using of a Laser Sheet Refractograms
}

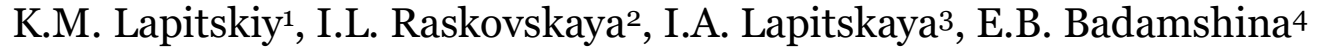 \\ National Research University «Moscow Power Engineering Institute»
}

1 ORCID: oooo-0oo2-4043-2049, LapitskyKM@mpei.ru

2 ORCID: 0ooo-0o02-1231-9479, RaskovskayaIL@mpei.ru

3 ORCID: oooo-ooo1-8924-3380, BamburkinaIA@mpei.ru

4 ORCID: oooo-0oo3-1181-7940, BadamshinaEB@mpei.ru

\begin{abstract}
The possibility of using the computer-laser refraction method for investigation of thermal inhomogeneities in liquids is demonstrated. A technique for direct task solution concerning refraction of a sheet laser beam on inhomogeneities of different structures is considered. The results of modeling the refraction of laser radiation applying the developed numerical algorithm for constructing the trajectories of geometric-optical rays in an inhomogeneous medium are presented. An experimental technique for reconstructing the temperature profile of inhomogeneity of liquid from refractograms is implemented.

Keywords: liquid inhomogeneitie, computer-laser refraction method, laser beam refraction, refractograms.
\end{abstract}

\section{Introduction}

The complexity of the experimental diagnostics of various liquid flows is mainly caused by their three-dimensional and unsteady nature, as well as the presence of significant gradients of all parameters that are of interest for practice. Under natural convection in heated bodies, the main thermophysical processes develop in a thin boundary layer several millimeters thick. In certain cases, physical properties of the liquid are also subject to significant changes in this layer [1], which must be considered when processing experimental data. The small thickness of the layer complicates the use of contact methods of experimental investigation. Consequently, the development of contactless optical methods for convection investigation and the comparison of experimental results with calculated data is highly relevant thermophysical task.

Contactless refraction methods for measuring temperature inhomogeneities in liquid [2 4] are characterized by comparative simplicity and visibility. Temperature gradients in liquid are visualized due to the deviation of the ray trajectory when passing through a medium with a variable refractive index.

The direct task of calculating the refraction of light on a temperature inhomogeneity in liquid is identical to calculating the transmission of a ray through an optical medium with a gradient change in the refractive index [5]. Whereby, the profile of the change of the refractive index along the ray trajectory differs from the standard optical models of gradient optics and is defined by a functional or numerical dependence on the spatial coordinate.

The inverse task of reconstructing the three-dimensional distribution of the temperature profile from refraction images, or refractograms can be solved within the framework of the adopted model of the temperature dependence on the refractive index of liquid.

In the present paper, smoothly inhomogeneous temperature fields are considered, in which the function of dependence of the refractive index on the coordinate does not have abrupt jumps and discontinuities. For cases when vortex flows arise in the medium [6], the 
solution of the inverse task of reconstructing the temperature profile is very difficult and in most cases impossible.

To solve the direct task of refraction on a temperature inhomogeneity in liquid, it is also possible to apply a numerical calculation method based on solving the equations of geometric optics. It allows investigating inhomogeneous media with a random functional temperature dependence. The present paper demonstrates the possibility of numerical calculation method's application for solving thermophysical tasks, as well as shows the possibility of solving the inverse task of reconstructing the temperature profile in an inhomogeneity with radial symmetry.

\section{Description of the experimental setup}

The principle of operation of computer-laser refraction (COLAR) method [2] is based on probing the flow with a laser sheet beam - an astigmatic beam with an elliptical cross-section, the size of which along one of the axes is significantly larger than the other. Such beam is also called a "laser sheet" (LS).

The setup (fig. 1) consists of a laser 1, an optical system for the formation of a LS 2, a cuvette with liquid 3 , a heated body 4 , a diffusing screen 5 , a digital video camera 6 , a personal computer 7 and a special software.

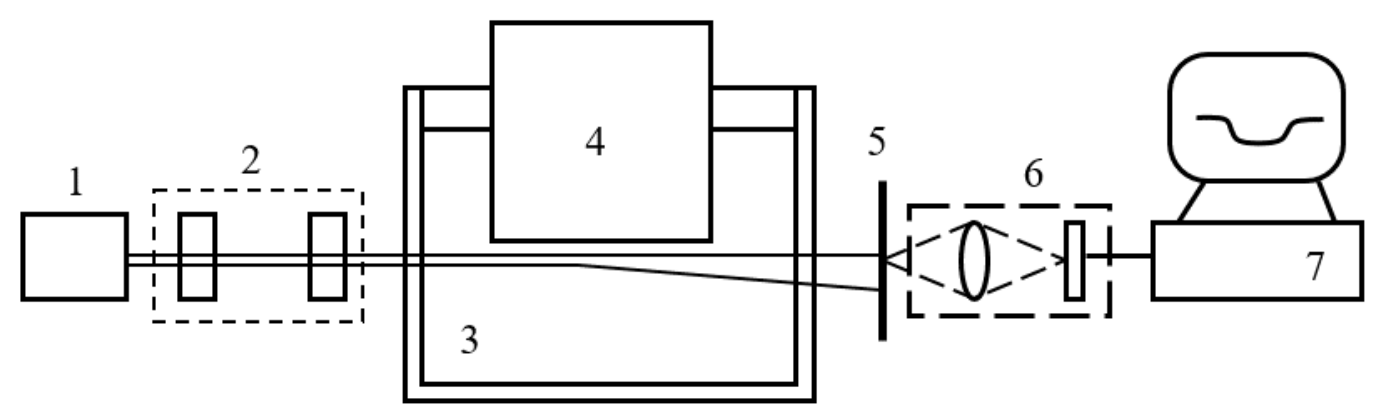

Fig. 1. Configuration of experimental setup of COLAR method

LS is directed under a heated object situated in a cuvette with liquid. During the LS propagation through an optically inhomogeneous flow, due to refraction, its particular areas deviate from the plane surface, i.e. a complex curved surface, changing both in space and in time, is obtained. LS projections at the exit from the optical inhomogeneity (refractograms) are observed on the screen, recorded by a digital video camera and entered into a computer for further processing.

Fig. 2 demonstrates examples of refractograms when a laser sheet beam passes through liquid near heated bodies of various shapes.

\section{Refraction of a laser sheet beam on a spherical inhomogeneity}

The example of an optical system for calculating a positive temperature inhomogeneity under a heated sphere is represented in fig. 3; the rectangle shows the liquid volume. The laser beam in the first approximation is replaced by a beam of rays, the deflection of which is shown in a vertical plane passing through the middle of the sphere in a vertical section. In the represented case, the temperature of the sphere $T_{b}$ was assumed to be higher than the temperature of liquid $T_{l}$. The calculated refraction curves conform with the coordinates of the beam on the right wall of the liquid volume, introduced according to the coordinate binding [7]. In the considered model of positive $\left(T_{b}>T_{l}\right)$ spherical temperature inhomogeneity, the dependence of the refractive index $n$ on temperature $T$ was calculated by the formula [2, 7]: 


$$
n(T)=1,3328-0,000051 T-0,0000011 T^{2} .
$$

Heated object under investigation
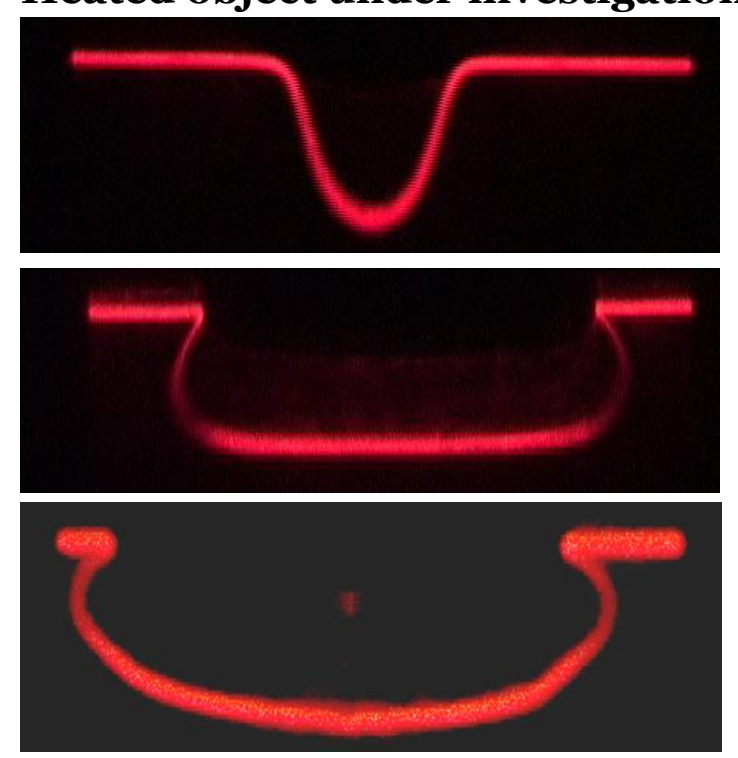

Fig. 2. Experimental refractograms of a laser sheet beam for various heated bodies in liquid

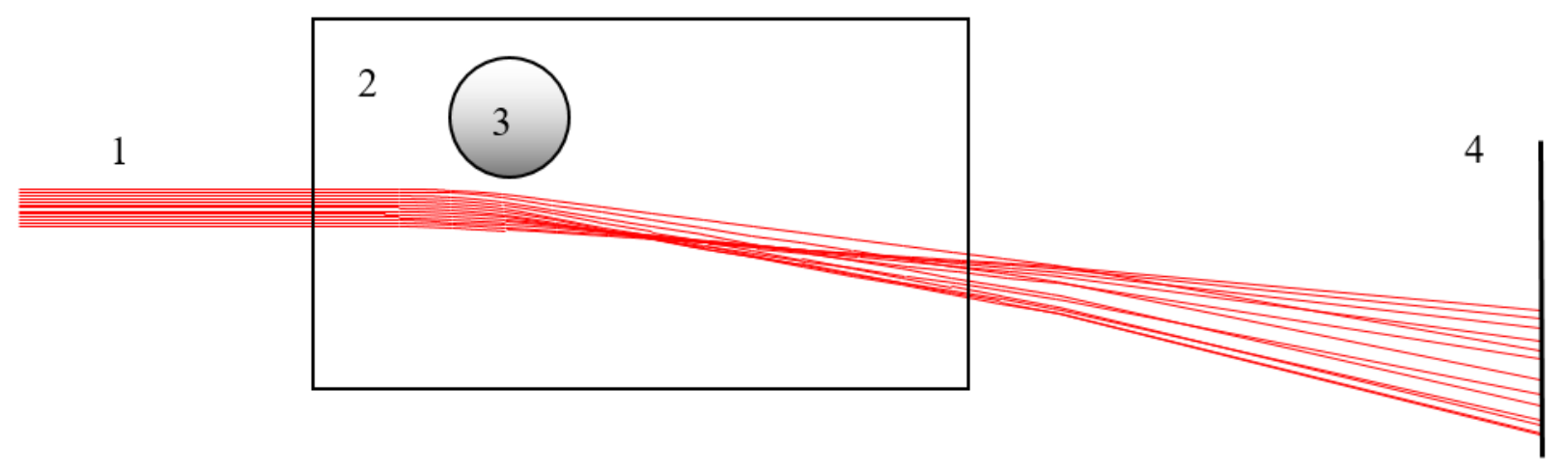

1 - laser beam, 2 - cuvette with liquid, 3 - heated sphere, 4 - screen

Fig. 3. Optical scheme for calculation of refraction in a temperature inhomogeneity under a heated sphere

For the heated sphere model the temperature $T_{b}=T$ was determined relative to the water temperature $T_{l}=T_{0}$, depending on the distance to the center of the sphere $r$ [7]:

$$
T(r)=T_{0}+\Delta T \exp \left[-\frac{(r-R-\Delta R)^{2}}{a^{2}}\right],
$$

where $T_{o}, \Delta T, \Delta R, a$ - temperature field model parameters. The parameter $T_{\mathrm{o}}$ is determined by the temperature of the walls of the cuvette with liquid, the value of $T(R)$ is equal to the temperature on the surface of the sphere, and the ratio $\Delta T / a$ corresponds to the gradient of the temperature field in the boundary layer with the thickness $a$. The value of the temperature gradient at $r=R$ is determined by the shift $\Delta R$, whereby the thermal conductivity of the sphere is not equal to the thermal conductivity of the liquid, there is a jump in the temperature gradient on the sphere surface and $\operatorname{grad}[T(r)] \neq 0$. However, the investigation does not exclude the possibility of equality or closeness to zero of the temperature gradient in the boundary layer (adhesion of the boundary layer), which determines the choice of the 
Gaussian model of the temperature field. Fig. 4 represents dependencies $T(r)$ and $n(r)$, plotted for the parameters $T_{0}=20^{\circ} \mathrm{C}, \Delta T=80^{\circ} \mathrm{C}, \Delta R=0, R=12 \mathrm{~mm}, a=1 \mathrm{~mm}$ at a section $R<r<r_{\max }$, where $r_{\max }=14,5 \mathrm{~mm}$, an approximate radius that is close to the asymptotic value $n\left(T_{0}\right)$.
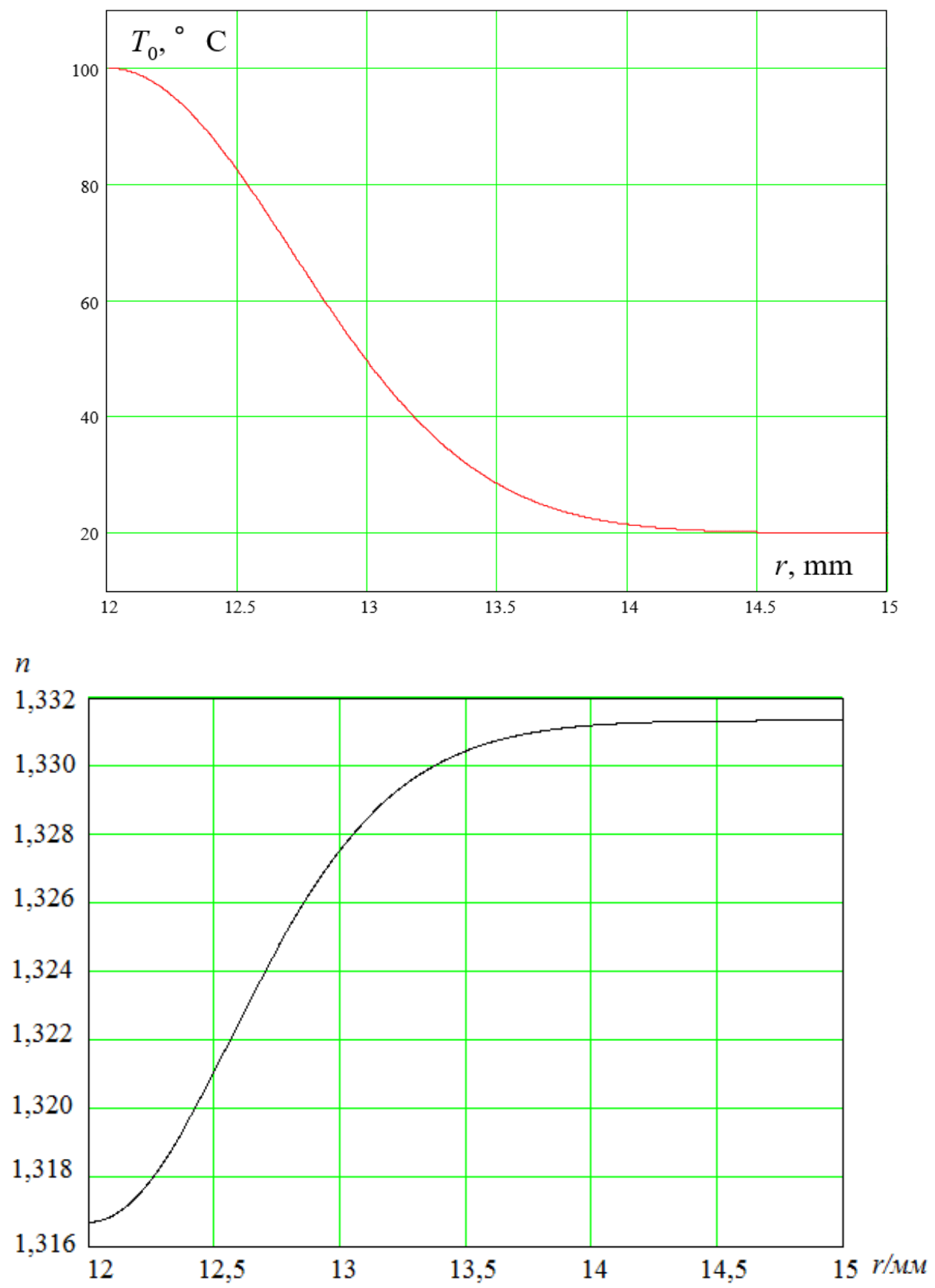

Fig. 4. Temperature profile and refractive index profile of water for the model: $R=12 \mathrm{~mm}, T_{0}=20^{\circ} \mathrm{C}, \Delta T=80^{\circ} \mathrm{C}, \Delta R=0, a=1 \mathrm{~mm}$ (approximation by the $5^{\text {th }}$ degree polynomials)

The calculated refraction patterns at a distance of $155 \mathrm{~mm}$ from the center of the sphere in the direction of beam propagation, obtained from analytical expressions and using a numerical algorithm, are demonstrated in fig. 5 . 


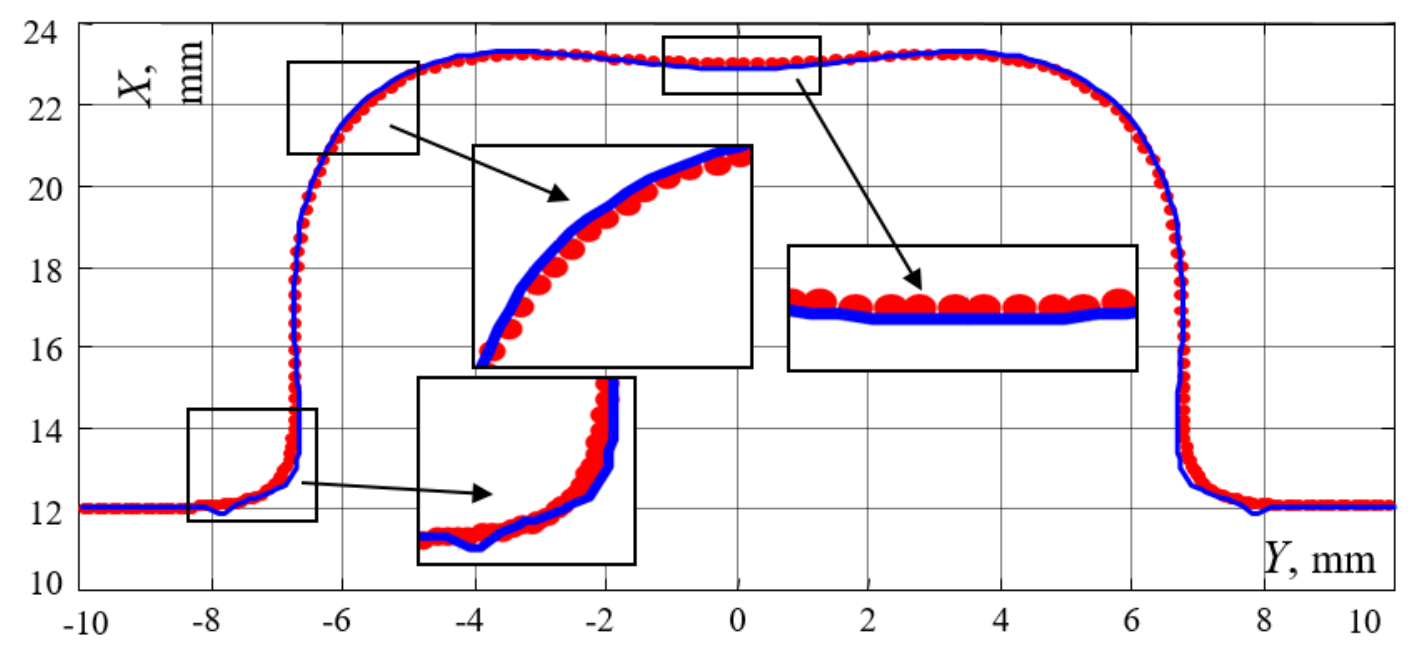

Fig. 5. Refraction curves for a heated sphere: $\bullet \bullet \bullet$ - analytical calculation, __ - calculation result CodeV

\section{Method for numerical calculation of refractograms}

Refraction methods for investigating optically transparent inhomogeneous media, based on measuring the deviation value of the probing radiation elements, allow high-quality visualization of the area under investigation. However, obtaining quantitative characteristics in most cases is difficult due to the complex spatial dependence of the refractive index of the medium. The analytical solution of both direct and inverse tasks can be obtained only for a plane-layered and radially-layered medium. In other cases, the solution of the direct task is possible only with the numerical methods application.

If the dependence of the refractive index on the coordinates $n(x, y, z)$ is known, then to find the equation of the ray trajectory, one can use the well-known differential equation [5], which makes it possible to determine the unitary vector $\mathbf{l}(x, y, z)$ tangent to the ray at each point of its trajectory:

$$
\frac{\mathrm{d}}{\mathrm{d} s}(n(x, y, z) \mathbf{l}(x, y, z))=\operatorname{grad} n \text {. }
$$

In [8], a numerical method for solving the reduced equation (3) is considered. The method is based on a step-by-step calculation of the trajectory of a ray passing through the area under investigation, and consists in finding a ray vector specified by the direction cosines in each section.

The computational region is divided into cells of size $\Delta z_{k}, \Delta x_{k}$ and $\Delta y_{k}$ (fig. 6), within which the value of the refractive index is considered constant: $n_{k}=n\left(x_{k}, y_{k}, z_{k}\right)$. The section of the trajectory within each cell is represented by a straight line. The method for choosing the size of the computational mesh cell is introduced in [8] and is based on solving test tasks that have an analytical solution.

The boundary condition of the problem is determined by the ray vector $\mathbf{l}_{\mathrm{o}}$ at the entrance to the inhomogeneous medium, namely, by its direction cosines $\left(\cos \alpha_{0}, \cos \beta_{0}, \cos \gamma_{0}\right)$ and the coordinate of the entrance to the inhomogeneous medium $\left(x_{0}, y_{0}, z_{0}\right)$.

The gradient of the refractive index in the kth cell is determined by the proportion $\operatorname{grad}_{k} n=\left.\operatorname{grad} n\right|_{x=x_{k}, y=y_{k}, z=z_{k}}$. Knowing $\mathbf{l}_{k}$, one can calculate the angle $\varphi_{k}$ between the unitary vector $\mathbf{l}_{k}$ and the vector $\operatorname{grad}_{k} n$ based on the scalar product: 


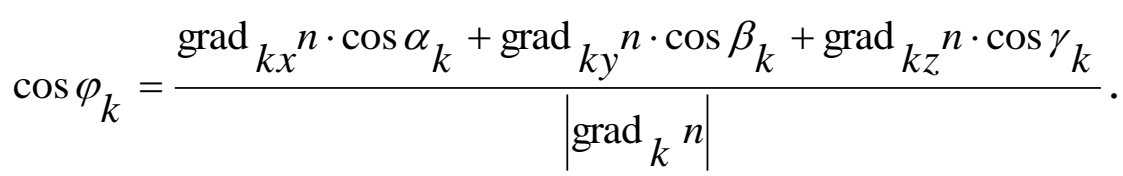

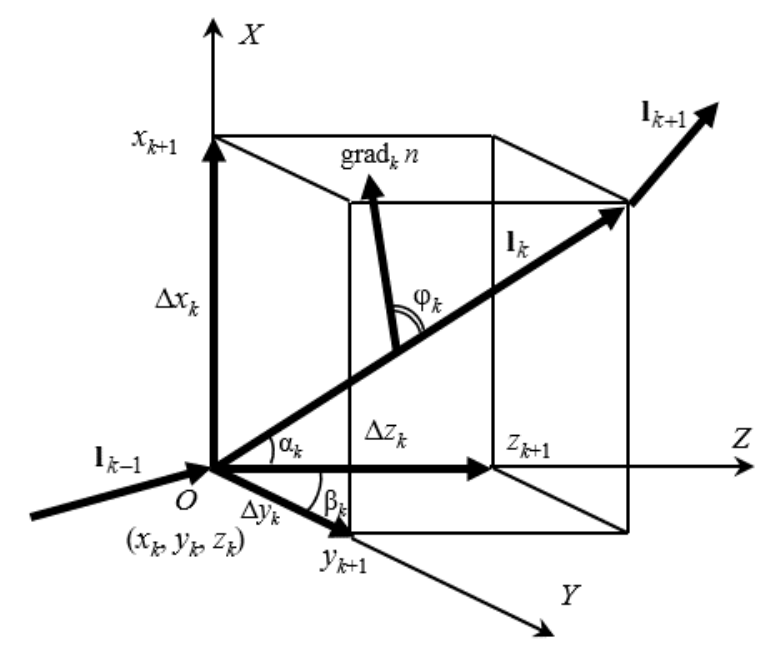

Fig. 6. Computational domain cell

The equation for the angle of rotation $\Delta \varphi_{k}$ of the vector $\mathbf{l}_{k}$ in the cell has the form [8]

$$
\Delta \varphi_{k}=\frac{\left|\operatorname{grad}_{k} n\right|}{n_{k}} \sin \varphi_{k} \sqrt{\left(\Delta x_{k}\right)^{2}+\left(\Delta y_{k}\right)^{2}+\left(\Delta z_{k}\right)^{2}} .
$$

The vector $\mathbf{l}_{k+1}$ in the next cell will equal $\mathbf{l}_{k+1}=\mathbf{l}_{k} \cos \Delta \varphi_{k}+\mathbf{v}_{k} \sin \Delta \varphi_{k}$, where $\mathbf{v}_{k}$ is the principal normal vector to the trajectory,

$$
\mathbf{v}=\frac{\operatorname{grad} n-\mathbf{l} \frac{\mathrm{d} n}{\mathrm{~d} s}}{|\operatorname{grad} n| \sin \varphi}
$$

As a result, at the exit from the computational region, we obtain the coordinates ( $\left.x_{\text {exit }}, y_{\text {exit }}, z_{\text {exit }}\right)$ and direction cosines $\left(\cos \alpha_{\text {exit }}, \cos \beta_{\text {exit }}, \cos \gamma_{\text {exit }}\right)$ of the ray vector $\mathbf{l}_{\text {exit. }}$ The refractogram at the exit from the optical inhomogeneity can be calculated based on specifying a family of rays at the entrance to the inhomogeneity.

\section{Refraction of a laser sheet beam on a cylindrical inhomogeneity}

The calculated refraction pattern of a laser sheet beam passing under the bottom of the cylinder is demonstrated in fig. 7. The optical inhomogeneity contains a qualitative edge effect observed in the experiment $[9,10]$, as a result of which the refraction pattern will have a strongly pronounced kink near the edge of the cylinder. The following parameters are accepted in the model: a cuvette with a thickness of $125 \mathrm{~mm}$, a cylinder in the middle, cylinder radius: $R=17 \mathrm{~mm}, \Delta R=-0,14 \mathrm{~mm}, a=0,7 \mathrm{~mm}, T_{0}=20^{\circ} \mathrm{C}, \Delta T=60^{\circ} \mathrm{C}$. The calculated curves are in satisfactory agreement with the test calculation using analytical expressions [9]. 


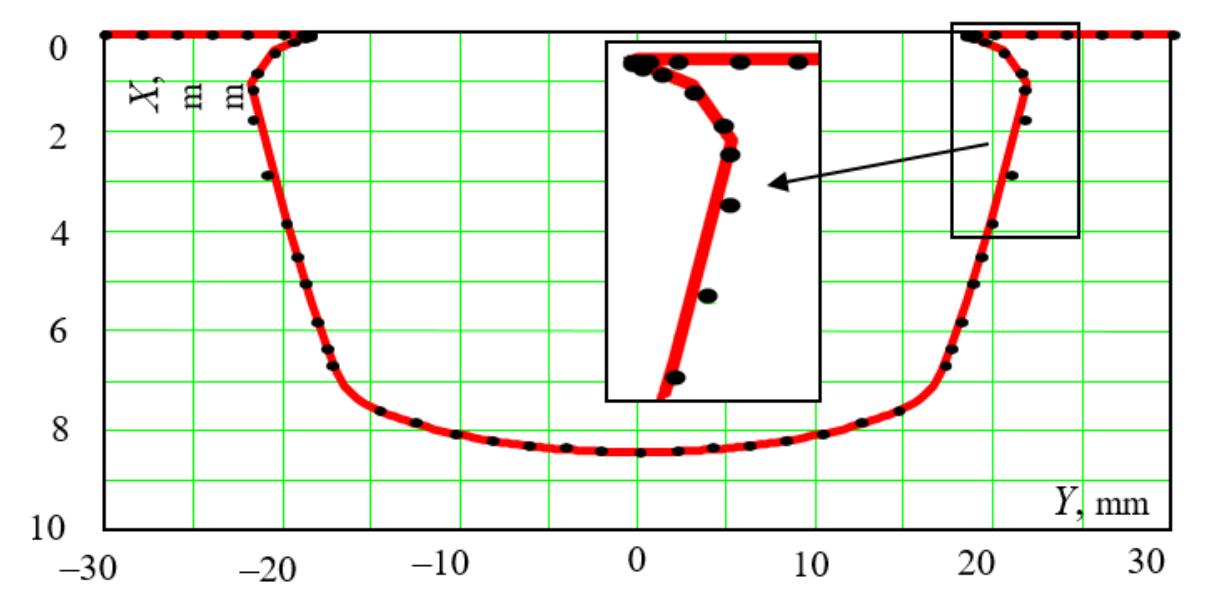

Fig. 7. Refraction curves for positive cylindrical inhomogeneity: $\bullet \bullet$ - numerical calculation, $\_$- calculation result CodeV

\section{Refractograms processing for spherical inhomogeneity}

The boundary layer in cold water around a heated body with a spherical structure (for example, a sphere or a cylinder with a hemispherical bottom) has a complex structure; however, in the region near the lower point of the hemisphere, the temperature field is spherical-layered with a high degree of accuracy. The deviation value of individual LS elements from the initial position depends on the temperature gradient along the line of propagation of the LS, while the deflection of the LS element occurs along a line passing through the center of spherical symmetry.

Let LS initially pass at a distance $h$ from the body. Let us consider an algorithm for processing refractograms in accordance with the scheme shown in fig. 8.

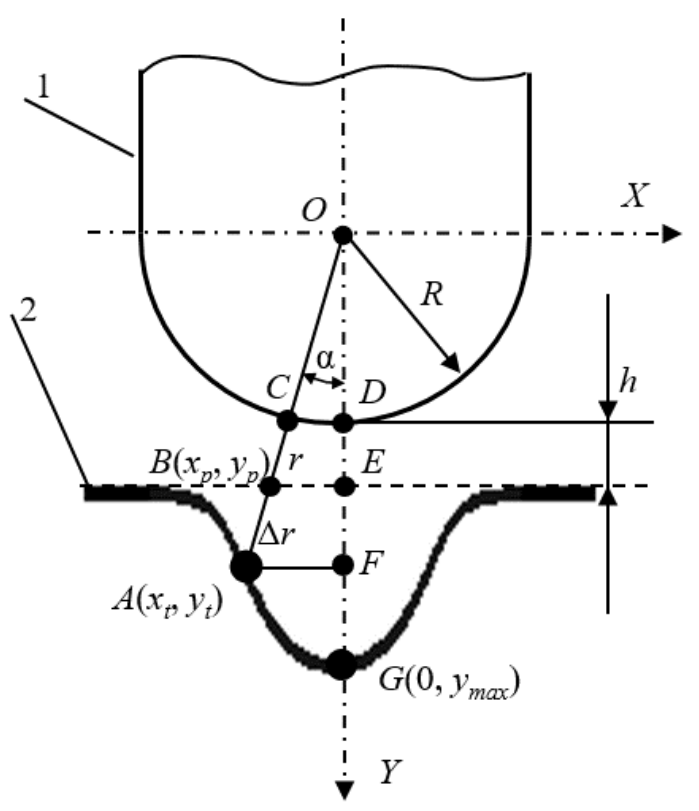

Fig. 8. Algorithm for processing refractograms

Let us briefly describe the stages [11]: 
1) All refractograms are preliminarily processed to plot the dependence of the maximum deviation of the LS element from the initial one along the $Y$ axis (point $G$ ) on time and body temperature.

2) An arbitrary point $A$ with coordinates $\left(x_{t}, y_{t}\right)$ of the refractogram is considered and the point of intersection of the straight line containing the segment $O A$ with the level $Y=R+h$ is found. It will be point $B$ with coordinates $x_{p}=(R+h) x_{t} / y_{t}, y_{p}=R+h$, which are obtained from considering the triangle $O A F$.

3) The value of deflection of the LS element along the selected direction equals to the length of the segment $A B=\Delta r=\left[\left(x_{t}-x_{p}\right)^{2}+\left(y_{t}-y_{p}\right)^{2}\right]^{1 / 2}$.

4) The value of $\Delta r$ is used to determine the temperature of the medium in a given direction at a distance $O B=r$ from the body surface according to the dependence constructed at stage 1 of the present algorithm. As a result, the dependence $T(r)$ is plotted.

It should be noted that in the experiment the temperature of the body surface is recorded, and not the temperature of the medium at a distance $h$ from the body. Therefore, to reduce the inaccuracy in the obtained distribution of the liquid temperature in the boundary layer, it is recommended to choose the value of $h$ as the smallest possible, which, as a rule, is determined by the size of the LS in the waist, which is located under the lower point of the hemisphere.

Fig. 9 demonstrates graphs of the dependence of the water temperature on the coordinate in the boundary layer based on the processing of refractograms (fig. 8) according to the mentioned algorithm for various temperatures $T_{\mathrm{c}}$ of the bottom of the cylinder.

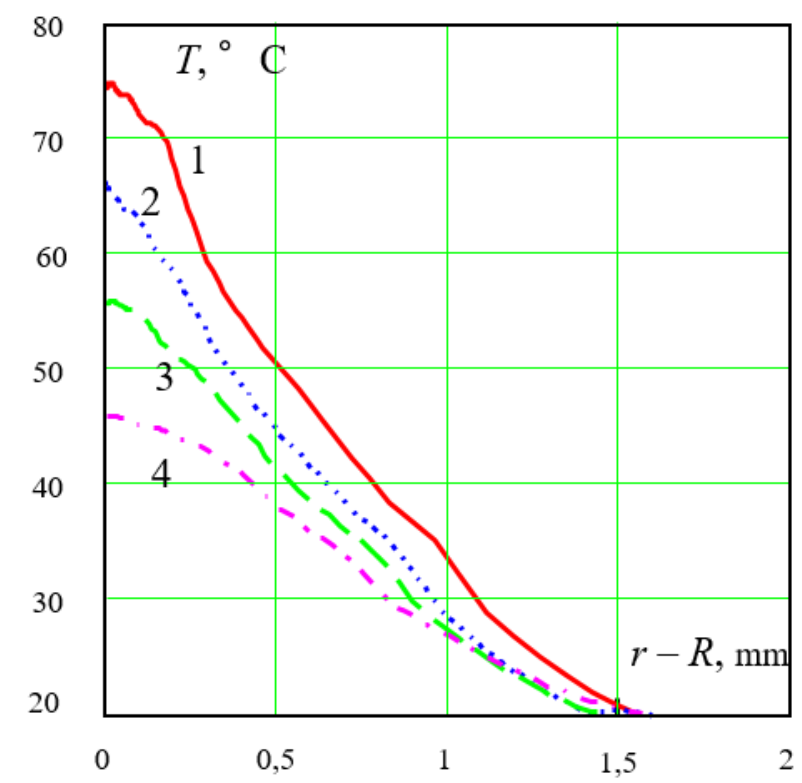

Fig. 9. Dependency of water temperature from coordinate in the boundary layer of the cylinder at the surface temperature:

$1-75{ }^{\circ} \mathrm{C}, 2-66{ }^{\circ} \mathrm{C}, 3-56^{\circ} \mathrm{C}, 4-46^{\circ} \mathrm{C}$

\section{Conclusion}

The results of calculating the refraction patterns of laser beams in thermal inhomogeneities in liquid, performed using a numerical algorithm, are in agreement with test calculations performed analytically and with a laboratory experiment. The presented computational algorithms are a reliable tool for solving direct and inverse tasks of natural convection by the laser refractography method. The appliance of the laser refraction method in the investigation of thermal fields in liquids also makes it possible to visualize graphically the structure of the temperature field. 


\section{References}

1. Polezhaev V.I. Convection and heat transfer in the near-critical area: research results in terrestrial and space conditions // Proceedings of RNKT-3. 2002. T.1. P.94-99.

2. Evtikhieva O.A., Raskovskaya I.L., Rinkevichius B.S. Laser refractography. - M.: FIZMATLIT, 2008. - 176 p.

3. Evtikhieva O.A., Raskovskaya I.L., Rinkevichius B.S., Tolkachev A.V. Fundamentals of laser refractography. // Modern optical methods for flows investigation: Multi-authored monograph. - M.: Overlay, 2011. P. $12-24$.

4. Artemov V.I., Yankov G.G., Evtikhieva O.A., Lapitskiy K.M., Raskovskaya I.L., Rinkevichius B.S., Tolkachev A.V. Numerical and experimental investigation of natural convection in liquid near a heated cylinder // Proceedings of the Fourth Russian National Conference on Heat Transfer. - M.: Publishing house MPEI, 2006. T.3. P. 42 - 46. 1980.

5. Kravtsov Yu.A., Orlov Yu.I. Geometric optics of inhomogeneous media. - M.: Science,

6. Varaksin A.Yu., Romash M.E., Kopeytsev V.N. Physical modeling of air tornadoes: issues of generation, stability and control. // Modern optical methods for flows investigation: Multi-authored monograph. - M.: Overlay, 2011. P. $252-274$.

7. Evtikhieva O.A. Refraction of the laser sheet in a spherically inhomogeneous thermal boundary layer // Measurement equipment. - 2006, № 5. P. 35 - 39.

8. Lapitskiy K.M., Raskovskaya I.L., Rinkevichius B.S. Algorithm for calculating refractograms of a laser sheet in an optically inhomogeneous medium. // Measurement equipment. - 2009, №5. P. $36-40$.

9. Tavrov A.V., Lapitskiy K.M. Calculation of the refraction of laser beams in liquids applying optical design programs // Proceedings of the 10-th International Scientific and Technical Conference "Optical Methods for Flows Investigation". / Edited by Yu.N. Dubnischev, B.S. Rinkevichius. - M.: Publishing house MPEI, 2009. P. 198 - 201.

10. Lapitskiy K.M., Raskovskaya I.L., Rinkevichius B.S. Calculation of refractograms in three-dimensional inhomogeneous media with edge effects // Proceedings of the 10-th International Scientific and Technical Conference "Optical Methods for Flows Investigation". / Edited by Yu.N. Dubnischev, B.S. Rinkevichius. - M.: Publishing house MPEI, 2009. P. 210 $-213$.

11. Lapitskiy K.M., Rinkevichius B.S., Tolkachev A.V. Quantitative visualization of natural convection near a heated body using a laser sheet // Proceedings of the 17-th School-seminar for young scientists and specialists under the guidance of. Academician of Russian Academy of Sciences A.I. Leontiev "Problems of gas dynamics and heat and mass transfer in aerospace technologies". - M.: Publishing house MPEI, 2009. T.1. P. 115 - 118. 